
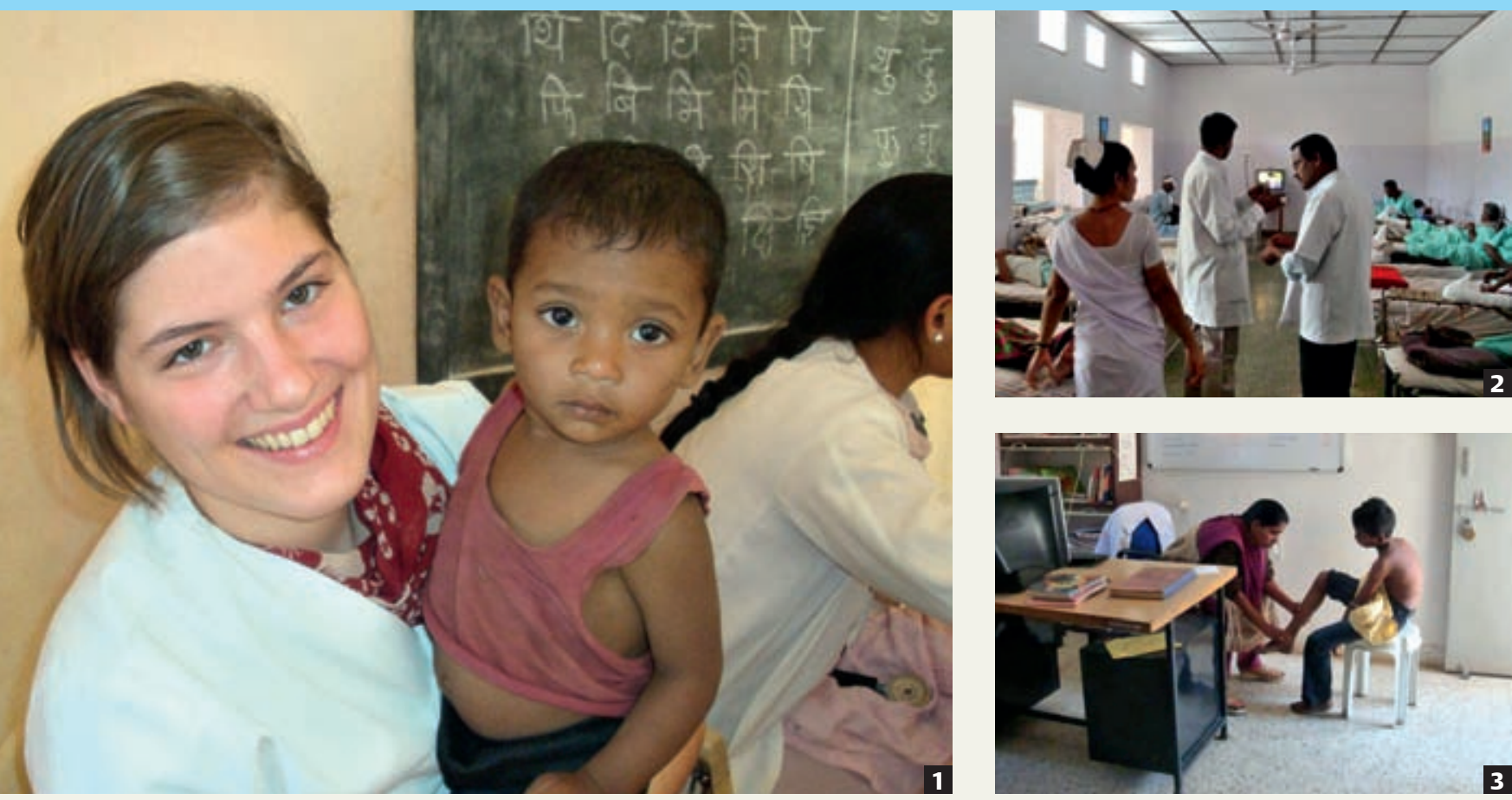

Abb. 1 Ans Herz gewachsen: Durch ihr Praktikum bei der „Internationalen Lepra-Mission“ in Indien lernte Daniela Ulmer viel über Land, Leute und Lepra. Der Abschied fiel ihr schwer, auch vom kleinen Samir.

Abb. 2 Visite auf der Männerstation: Frauen und Männer sind in Indien auf getrennten Fluren untergebracht, den Männern ist das Betreten der Frauenstation verboten. In Kothara hat es auf beiden Stationen jeweils Platz für 20 Patienten.

Abb. 3 Das A und O: Da weiße Hautareale die ersten Anzeichen für Lepra sind, wird bei jedem Patienten in Kothara erst einmal die Haut untersucht - ganz egal, wegen welcher Beschwerden die Patienten die Klinik aufsuchen.

Abb. 4 Krafttest des M. abductor pollicis longus: Die Physiotherapeuten müssen für den Leprabefund die Muskelfunktionstests für jeden noch so kleinen Muskel an Händen, Füßen und im Gesicht parat haben.

Abb. 5 Neu und strahlend weiß: So sieht der Gips von Akash nicht mehr aus, wenn er in sechs Wochen zur Nachbehandlung kommen wird. Denn mit dem eingearbeiteten Metallstück ist das Laufen kein Problem, sodass auch die Feldarbeit weitergehen kann. 


\title{
Die vergessene Krankheit
}

\author{
ERFAHRUNGEN IN EINER LEPRA-MISSION Was wissen wir heutzutage \\ in Europa noch über Lepra? Diese Krankheit ist vergessen und vorbei - \\ außer man besucht Länder, die nach wie vor damit zu kämpfen haben. \\ Daniela Ulmer hat das gemacht. Sie berichtet von ihrem Praktikum auf \\ einer Station der „Internationalen Lepra-Mission“ im indischen Kothara.
}

A utos, Motorräder, Pferdewagen, Fußgänger und frei herumlaufende Kühe teilen sich eine Straße. Kinder spielen am Straßenrand, wo Obst- und Gemüsehändler neben Müllbergen ihre Waren anpreisen in Indien kommt ein Europäer aus dem Staunen nicht mehr heraus. Genau darum hat mich dieses Land schon immer gereizt. Kurz nach meinem Physiotherapieexamen bewarb ich mich bei der Entwicklungsorganisation „Internationale Lepra-Mission“ für ein Praktikum in einem indischen Leprakrankenhaus. Ich wollte Land, Leute und vor allem die Behandlung einer Krankheit kennenlernen, von welcher wir in Europa kaum mehr etwas wissen. So verbrachte ich acht Wochen in Kothara, einer Kleinstadt im Herzen Indiens, rund fünf Autostunden vom nächsten Inlandsflughafen entfernt. Nur eine einzige geteerte Straße führt dorthin.

Erste Anzeichen: Helle Hautflecken > Die Klinik ist eines von vielen Projekten der „Internationalen Lepra-Mission“. Mittels Spendengelder unterstützt sie weltweit von Lepra betroffene Menschen. Der indische Staat finanziert Aufklärungsprogramme und Operationen. So ist die Behandlung für Patienten mit Lepra kostenlos. Aus eigener Tasche könnten ohnehin die wenigsten die Therapie zahlen, denn Lepra ist nach wie vor die Krankheit der Armen. Sie wird über das Mycobacterium leprae vermutlich per Tröpfcheninfektion übertragen. Die ersten Anzeichen sind weiße Hautareale mit Sensibilitätsstörungen. Im Verlauf kommt es zu Nervenschädigungen, die wiederum Lähmungen und in Folge Gelenkkontrakturen verursachen. Durch die gestörte Sensibilität verletzen sich die Patienten oft, was zu
Wundinfektionen oder gar einem Ulkus führen kann. Schlimmstenfalls droht ihnen eine Amputation. Doch dank verschiedener Therapien kommt es dazu heutzutage selten.

Kothara ist aber nicht nur Anlaufstelle für Patienten mit Lepra. Allen, denen es möglich ist zu kommen, steht die Klinik offen. So finden auch Patienten mit orthopädischen (Arthrosen, Frakturen) und neurologischen Beschwerden (Schlaganfällen) den Weg in die Klinik. Der Schwerpunkt liegt dennoch auf der Therapie von Lepra. Auf diesem Gebiet sind alle 54 Mitarbeiter

\section{Verstümmelung heißt Ausgrenzung.}

speziell ausgebildet. Ein Allgemeinarzt, ein Dermatologe, eine Augenärztin und eine Internistin bieten eine für Indien breite medizinische Abdeckung. Für Eingriffe reisen Chirurgen aus anderen Kliniken der „Internationalen Lepra-Mission“ an. Die Eckpfeiler der Lepratherapie sind die Prävention, medizinische Hilfe und Rehabilitation. Außerdem können die Patienten und ihre Familien an Gesprächs- und Seelsorgerunden teilnehmen, da sich die Wiedereingliederung in das familiäre Umfeld oft schwierig gestaltet: Eine Verstümmelung ist Grund zur Ausgrenzung, da der Betroffene die Familie nicht mehr unterstützen kann.

Arztbesuch: Familie kommt mit > Für den europäischen Gaumen sind die indischen Speisen sehr pikant. Mit Weizen- oder Kartoffelfladen, einem würzigen TomatenChutney und Chai-Tee als Frühstück startete ich in den Tag. Danach trafen sich alle Mitarbeiter zu einer kleinen Andacht in der Kapelle. Um halb neun begannen Ärzte, Pflegekräfte und Physiotherapeuten mit der Visite (॰ Abb. 2). Die Stimmung war heiter, das Tempo von Bett zu Bett gemütlich, auch wenn es voll war.

Im Anschluss daran gingen wir in die Ambulanz. Dort warteten meist schon frühmorgens viele Patienten mit ihren Verwandten. Sie haben im Morgengrauen ihre Hütten verlassen, um noch vor der Hitze in die Klinik zu marschieren. Andere, deren Anreise den ganzen Tag dauerte, trafen erst am Abend völlig erschöpft in der Ambulanz ein. Jeder kommt nach seinen Möglichkeiten und zu seiner Zeit zum Arzt - Überweisungen und ein ambulantes System wie in Deutschland gibt es nicht.

Befund: Hemdzuknöpfen auf Zeit > Egal wegen welchen Beschwerden die Patienten die Ambulanz aufsuchen, bei allen wird zuerst die Haut auf Pigmentveränderungen untersucht. Diese hellen Flecken sind auf der dunklen Haut der Inder leicht zu erkennen. Fanden wir weiße Stellen, brachte eine Blutuntersuchung Klarheit darüber, ob sie auf Lepra zurückzuführen sind. Bei den Leprakranken diente uns der Hautbefund zur Kontrolle, ob die Medikamente anschlagen. Zudem untersuchten wir ihre betroffenen Extremitäten, was meine Hauptaufgabe in der Ambulanz war. Für die Befunde gab es elektronische Dokumentationsformulare, in denen ich die Ergebnisse der Sensibilitäts- und Muskelkrafttests speicherte. Im Gesicht, an Händen und Füßen musste ich jeden Muskel einzeln testen ( $\odot$ Abb. $\mathbf{3}$ und 4) - die indischen Physiotherapeuten legten 
